\title{
Immunotherapy in Ovarian Cancer: Thinking Beyond PD-1/PD-L1
}

\author{
Laure Chardin $^{1}$ and Alexandra Leary ${ }^{1,2 *}$ \\ 1 Université Paris-Saclay, Institut Gustave Roussy, Inserm U981, Biomarqueurs Prédictifs et Nouvelles Stratégies \\ Thérapeutiques en Oncologie, Villejuif, France, ${ }^{2}$ Department of Medical Oncology, Université Paris-Saclay, Institut Gustave \\ Roussy, Inserm U981, Biomarqueurs Prédictifs et Nouvelles Stratégies Thérapeutiques en Oncologie, Villejuif, France
}

Ovarian cancer $(\mathrm{OC})$ is the most lethal gynecologic malignancy, affecting approximately 1 in 70 women with only $45 \%$ surviving 5 years after diagnosis. This disease typically presents at an advanced stage, and optimal debulking with platinum-based chemotherapy remains the cornerstone of management. Although most ovarian cancer patients will respond effectively to current management, $70 \%$ of them will eventually develop recurrence and novel therapeutic strategies are needed. There is a rationale for immune-oncological treatments $(\mathrm{IO})$ in the managements of patients with $\mathrm{OC}$. Many $\mathrm{OC}$ tumors demonstrate tumor infiltrating lymphocytes (TILS) and the degree of TIL infiltration is strongly and reproducibly correlated with survival. Unfortunately, results to date have been disappointing in relapsed $\mathrm{OC}$. Trials have reported very modest single activity with various antibodies targeting PD-1 or PD-L1 resulting in response rate ranging from $4 \%$ to $15 \%$. This may be due to the highly immunosuppressive TME of the disease, a low tumor mutational burden and low PD-L1 expression. There is an urgent need to improve our understanding of the immune microenvironment in $\mathrm{OC}$ in order to develop effective therapies. This review will discuss immune subpopulations in OC microenvironment, current immunotherapy modalities targeting these immune subsets and data from clinical trials testing $\mathrm{IO}$ treatments in $\mathrm{OC}$ and its combination with other therapeutic agents.

Keywords: ovarian cancer, immunotherapy, PD-L1, immunosuppression, tumor microenvironment

\section{INTRODUCTION}

Ovarian cancer (OC) is the most frequent cause of death among gynecological malignancies, with a 36\% increase in OC incidence being expected by 2040 (source Global Cancer Observatory 2020). Optimal debulking followed by platinum-based chemotherapy remains the cornerstone of management (1). For patients with bulky stage III-IV tumors where complete resection cannot be achieved, neo-adjuvant chemotherapy followed by interval debulking surgery and adjuvant chemotherapy is a suitable alternative associated with lower morbidity $(2,3)$. Unfortunately, despite a response rate of $80 \%$ to first line chemotherapy, most patients subsequently relapse resulting in a five year survival rate of $45 \%$ (4). To enhance long-term disease remission, new treatment approaches are under investigation.

Immunotherapy has demonstrated great potential in treating a variety of cancers, in particular immune checkpoint inhibitors (ICI) targeting cytotoxic T-lymphocyte-associated protein 4 (CTLA-4) 
or Programmed death-ligand 1 (PD-L1)/Programmed cell death protein $1(\mathrm{PD}-1)(5,6)$. There is a rationale for immune-oncology (IO) treatments in OC. Many OC tumors demonstrate tumor infiltrating lymphocytes (TILs) and the degree of TIL infiltration is strongly and reproducibly correlated with survival $(7,8)$. A metaanalysis including 21 studies and almost 3000 patients with OC confirmed that high levels of intra-epithelial $\mathrm{CD}^{+}$or $\mathrm{CD}^{+} \mathrm{T}$-cells were most strongly associated with both improved progressionfree and overall survival (PFS and OS) (9). This positive correlation suggests that using ICIs, such as anti-PD-L1/PD-1 therapies, could be effective. Contrary to expectations, early clinical trials showed that their efficacy in OC remains limited with response rate of $10-15 \%$ and no current FDA or EMA approval (Table 1). PD-L1 expression has emerged as one of the biomarkers that could predict sensitivity to ICIs (15). Unfortunately expression remains rare in OC. PD-1 is mainly expressed by $\mathrm{CD}^{+}$and $\mathrm{CD}^{+} \mathrm{T}$ lymphocytes whereas its ligand, PD-L1 is widely expressed in various cell types including activated lymphocytes, fibroblasts, tumor-associated macrophages, and tumor cells. A study showed that almost two thirds of ovarian tumors demonstrated a modest expression of PD-L1 which was associated with worst prognosis, mainly on immune cells rather than tumor cells. In the IMAGYN050 trial, less than $25 \%$ of patients demonstrated $>5 \%$ PD-L1+ immune cells (16). In contrast, in tumors known to be immune responsive, such as non-small cell lung cancer, PD-L1 expression ranges from $24 \%$ to $60 \%$ (17). The low response rate to PD-L1 inhibition in OC could be in part explained by the low expression level of PD-L1 on tumor cells.

Several studies have confirmed the relevance of using mutational and tumor antigen burden as a predictive biomarker of response to immunotherapy (18-21). However, OC is known to harbor a low neoantigen load and mutational burden (22-24). There is some data to suggest that BRCA mutated, or homologous recombination deficient OC may harbor higher levels of 'personal' neoantigens presumably due to their defective DNA repair machinery (25). In addition, OC have been reported to demonstrate cancer associated antigens such as NY-ESO-1, mutated p53, Mesothelin, MUC-16, SCP-1... which could drive immunogenecity (26). In this review we will discuss the ongoing strategies which are being explored to enhance the antitumor immune response in OC beyond PD-1/ PD-L1 inhibition including: (i) combining anti PD-1/PD-L1 agents with other agents, and (ii) targeting other relevant immune subsets.

\section{COMBINATION APPROACHES}

\section{Poly (ADP-Ribose) Polymerase (PARP) Inhibitors and ICls}

Approximately 25\% of high grade serous ovarian cancer harbor a germline or somatic mutations in the tumor suppressor genes BRCA1 or BRCA2 $(27,28)$. BRCA1/2 mutated OC are associated with a higher lymphocyte infiltration $(25,29)$. BRCA1/2 are involved in DNA damage response via the homologous recombination (HR) pathway. HR participates in genome stability by repairing complex DNA damage such as DNA double-stranded breaks. Several studies have highlighted that $B R C A 1 / 2$ mutated OC harbor a higher number of tumor specific neo-antigenes and demonstrate increased expression of the immune checkpoint modulators, PD-1 and PD-L1, which indicated that BRCA1/2 mutated OC may be more sensitive to PD-1/PD-L1 inhibitors (25). Unfortunately, the JAVELIN 100 trial assessing the efficacy of avelumab (anti-PD-L1) in patients with previously treated recurrent of refractory OC showed that BRCA status was not associated with clinical response (30).

Recently, increasing evidence has suggested the importance of the link between DNA damage and innate immunity (31). PARP inhibition in Brcal-deficient mouses elicits strong antitumor immunity via Stimulator of Interferon Genes (STING) pathway activation (32). PARPi induced STING activation occurs mainly in tumor cells. This pathway results in release of interferon related cytokines which in turn increase NK and other cell mediated cell killing via upregulation in NKG2D ligand for example. The MEDIOLA trial evaluated the combination of Olaparib (PARPi) and Durvalumab (anti-PD-L1) in BRCA mutated platinum-sensitive relapsed OC (33). The objective response rate was high at $71,9 \%$ but should be interpreted with caution as this response rate could be expected with a PARPi alone in $B R C A$ altered OC. It is therefore difficult to conclude

TABLE 1 | Results from trials exploring efficacy and safety of single-agent ICls in OC.

\begin{tabular}{|c|c|c|c|c|c|c|}
\hline Study & Trial identifier & $\begin{array}{l}\text { Number } \\
\text { of } \\
\text { patients }\end{array}$ & ORR & $\begin{array}{c}\text { PFS } \\
\text { (Months) }\end{array}$ & $\begin{array}{c}\text { OS } \\
\text { (Months) }\end{array}$ & Ref \\
\hline $\begin{array}{l}\text { Efficacy and Safety of Avelumab for Patients With Recurrent or Refractory Ovarian Cancer: Phase 1b } \\
\text { Results From the JAVELIN Solid Tumor Trial }\end{array}$ & NCT01772004 & 125 & $9.6 \%$ & 10.2 & 11.2 & $(10)$ \\
\hline $\begin{array}{l}\text { A Study of Atezolizumab [an Engineered Anti-Programmed Death-Ligand } 1 \text { (PDL1) Antibody] to } \\
\text { Evaluate Safety, Tolerability and Pharmacokinetics in Participants With Locally Advanced or } \\
\text { Metastatic Solid Tumors }\end{array}$ & NCT01375842 & 12 & $22.2 \%$ & 2.9 & 113. & $(11)$ \\
\hline Phase IB Study of Pembrolizumab (MK-3475) in Subjects With Select Advanced Solid Tumors & NCT02054806 & 26 & $11.5 \%$ & 1.9 & 13.8 & $(12)$ \\
\hline $\begin{array}{l}\text { Efficacy and Safety Study of Pembrolizumab (MK-3475) in Participants With Advanced Recurrent } \\
\text { Ovarian Cancer (MK-3475-100/KEYNOTE-100) }\end{array}$ & NCT02674061 & 376 & $8.0 \%$ & 1.9 & 13.8 & (13) \\
\hline $\begin{array}{l}\text { Safety and Antitumor Activity of Anti-PD-1 Antibody, Nivolumab, in Patients With Platinum-Resistant } \\
\text { Ovarian Cancer }\end{array}$ & UMIN00005714 & 20 & $15 \%$ & 3.5 & 20.0 & $(14)$ \\
\hline
\end{tabular}


that there was synergistic or even additive benefit to this combination.

Combination of PARPi and anti-PD-L1 has also been tested in BRCA wild type OC. The combination of the PARPi niraparib and pembrolizumab (anti-PD-1) resulted in an encouraging $25 \%$ $\mathrm{RR}$ among patients with mainly platinum resistant BRCAwt recurrent OC (34).

\section{Anti-Angiogenic Agents and ICls}

Vascular endothelial growth factor (VEGF) is a key regulator of physiological and pathological angiogenesis and plays a major role in tumorigenesis (35). VEGF is highly expressed in OC microenvironment (36). It promotes tumor angiogenesis, enhances vascular permeability and favors peritoneal dissemination of OC through malignant ascites formation (37). In addition to its contribution to tumor angiogenesis, VEGF also has immunosuppressive properties. VEGF inhibits T-cell function, contributes to the induction and maintenance of regulatory $\mathrm{T}$ cells (Tregs), inhibits functional maturation of DC, enhances expression of inhibitory immune checkpoint on $\mathrm{CD}^{+}$cells and promotes tumor-associated macrophages (38). Combining anti-angiogenic agents with ICIs could reverse immunosuppression mediated by VEGF and thus increase the efficacy of ICIs in OC. In vitro, VEGF inhibition has been shown to enhance cytotoxic T-lymphocytes activation and downregulate inhibitory molecules associated with $\mathrm{T}$ cell exhaustion (PD-L1, TIM-3, LAG-3 and CTLA-4 $(39,40)$.

A single-arm phase 2 study of combined nivolumab and bevacizumab resulted in a $40 \%$ and $16 \%$ response rate in platinum-sensitive and -resistant relapsed OC (41). Combination of the VEGF tyrosine kinase, Lenvatinib with pembrolizumab resulted in response rate of $29 \%$ in relapsed OC (42). Despite a sound biological rational and hints of activity in early phase trials of combined PD-L1/VEGF inhibition, a large phase III randomized clinical trial of $1^{\text {st }}$ line chemotherapy and maintenance bevacizumab alone or in combination with atezolizumab failed to demonstrate any benefit to the combination (16).

\section{Chemotherapy and ICls}

Classical cytotoxic drugs have been shown to alter the local immune state which could modulate treatment efficacy by stimulating or inhibiting the host's anti-tumor immune response (43-45). Conventional cytotoxics may induce " immunogenic cell death ", increase DC maturation, potentiate macrophage cytotoxicity and abrogate Tregs or myeloid-derived suppressor cell activity $(46,47)$. Two phase III clinical trials evaluated the combination of avelumab (anti-PD-L1) to standard chemotherapy and failed to show any improvement of avelumab addition in the frontline (30) or in the platinum resistant setting (48).

\section{Anti-CTLA-4 and ICls}

CTLA-4 is a receptor expressed on activated $\mathrm{T}$ cells that downregulate immune response. CTLA- 4 is homologous to the T-cell co-stimulatory protein, CD28, and both molecules binds to CD80 and CD86, two co-stimulatory molecules expressed on antigen-presenting cells. Interactions of these ligands with
CTLA-4 inhibits T-cell activation. Blockade of CTLA-4 with anti-CTLA-4 antibodies enhances priming and activation of naïve $\mathrm{T}$-cells in lymph nodes and then migrate to tumors to cause tumor rejection. Emerging evidences suggested that combined PD-L1/PD-1 and CTLA-4 blockade could be relevant in OC. NRG GY003, a phase II trial evaluating nivolumab alone or in combination with ipilimumab, demonstrated a higher response rate $(31.4 \%)$ with the combination compared to nivolumab alone (12.2\%) (49). Various ongoing trials are evaluating the benefit of anti-PD-1/PD-L1 and CTLA-4 therapy including the IneOV (NCT03249142) trial which is evaluating the combination of neoadjuvant Durvalumab and chemotherapy +/- Tremelimubab (anti-CTLA-4) in 66 patients with inoperable OC. Preliminary results showed that the combination achieved an overall macroscopic complete resection rate of $58 \%$ and a rate of major pathological response (Chemotherapy Response Score 3) of 38\%. However, addition of Tremelimumab did not increase CCO or CRS3 rates $(50)$.

\section{IMMUNOSUPPRESSION IN OVARIAN CANCER}

PD-L1/PD-1 inhibition in ovarian cancer remains disappointing. $\mathrm{CD}^{+}$cells and PD-L1 may not be the only relevant immune targets in OC. Other immune subsets such as tumors-associated macrophages (TAMs), cancer-associated fibroblasts (CAFs) or regulatory $\mathrm{T}$ lymphocytes (Tregs) may be crucial in mediating immune tolerance and resistance (Figure 1) to $\mathrm{PD}-\mathrm{L} 1 / \mathrm{PD}$ 1 inhibition.

\section{Tumor-Associated Macrophages}

Among the numerous factors that play a pivotal role in immunosuppressive TME of OC, TAMs are the most abundant infiltrating immune cells, particularly in malignant ascites (51-53). Due to their plastic nature, macrophages may polarize into two distinct forms depending on the local environment, the antitumorigenic (M1-like) and the pro-tumorigenic (M2-like).

In the OC microenvironment, TAMs generally exhibit the M2like phenotype, with high expression of scavenger receptor class $\mathrm{B}$ (CD163), mannose receptor (CD206) and immunosuppressive factors, including interleukin-10 (IL-10), IL-6, TGF- $\beta$, as well as chemokines CCL18 and CCL22 to support immune escape and angiogenesis (54-56). A high density of CD163+ M2macrophages is associated with poor prognosis in epithelial ovarian cancer whereas high ratio of $\mathrm{M} 1 / \mathrm{M} 2$ was associated with extended survival in OC patients $(57,58)$.

TAMs induce an immunosuppressive environment that suppresses the function of T cells, DCs, and natural killer (NK) cells and activates the function of regulatory T cells (Tregs) (5961). TAMs can suppress T-cell activity by the depletion of Larginine in the tumor microenvironment. Indeed, the expression of Arginase 1 by TAMs leads to the depletion of L-arginine which is essential for T-cell functions and TCR signaling (62-64). Binding of SIRP $\alpha$ expressed on the surface of myeloid cells to its ligand CD47 on tumor cells acts as a "don't eat me signal » 


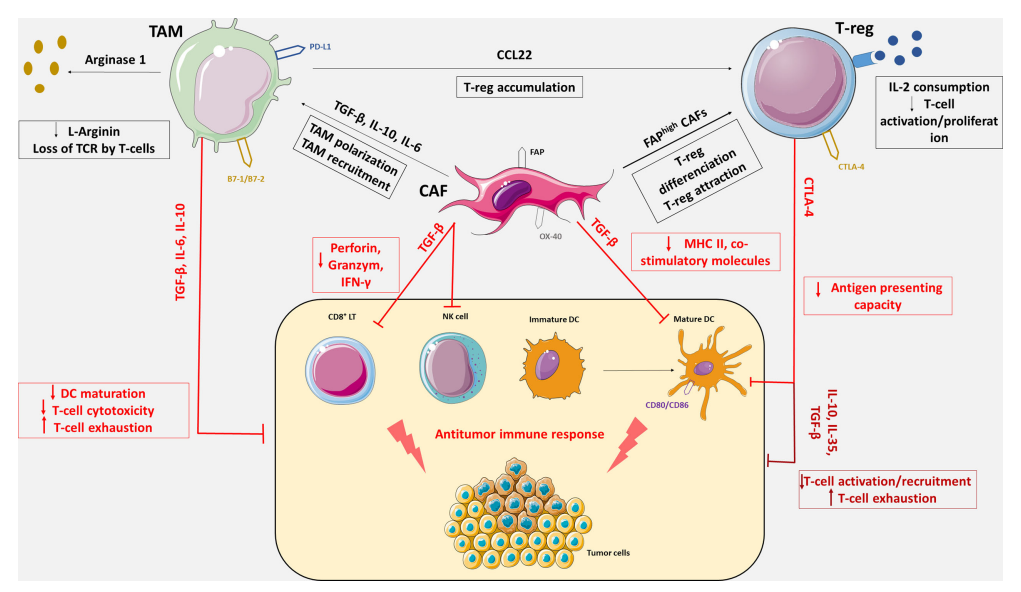

FIGURE 1 | Immunosuppressive tumor microenvironment mediated by Tregs, CAFs and TAMs. OC tumor microenvironment includes antitumor immune cells such as cytotoxic CD8 ${ }^{+}$T lymphocytes (CD8 ${ }^{+} \mathrm{LT}$ ), natural killer cells (NK cells) and dendritic cells (DC), and immune tolerant cells such as tumor associated macrophages, cancer associated fibroblasts and regulatory $T$ cells responsible for immune escape. TAMs, CAFs and Tregs express an array of effector molecules that inhibit the antitumor immune responses including cell surface receptors, cytokines, chemokines, and enzymes. Through the expression of immunosuppressive cytokines including TGF- $\beta, I L-6, I L-10$ and IL-35, TAMs, CAFs and Tregs inhibit CD8 ${ }^{+}$LT recruitment, activation and cytotoxicity, promote CD8 ${ }^{+}$LT exhaustion and impede DC maturation. CAFs also reduce antigen presentation function of DC via the secretion of TGF- $\beta$, which downregulate the expression of MHC II and co-stimulatory molecules on DC. CAFs can secrete IL- 6 and thereby contribute to monocytes recruitment and macrophages differentiation to M2-like phenotype. TGF- $\beta$ expression by CAFs negatively regulate NK cells activation and cytotoxic activity. FAPhigh CAFs increase differentiation of CD4 ${ }^{+}$cells into CD25 ${ }^{+}$FoxP3 ${ }^{+}$Tregs and retain them at their surface by expression of OX-40. Tregs constitutively express the co-inhibitory molecule, CTLA-4 which inhibits antigen presentation by binding on CD80 and CD86, co-stimulatory molecules expresses on DC. Tregs also inhibit CD8+ LT activation via IL-2 consumption which is necessary to T-cells activation. The cytokine CCL22 produces by TAMs generate chemokine gradient that induces Treg accumulation in the TME. TAMs also express co-inhibitory molecules such as PD-L1 or B7-1/B7-2 and suppress CD8 ${ }^{+}$LT cytotoxic activity upon activation with their ligand, PD-1 and CTLA-4. TAMs also impair LT activity though metabolization of LArginine which is essential for T-cell function and TCR signaling.

(65). CD47 expression in patients with OC correlates with poor prognosis, potentially by inhibiting macrophage phagocytosis $(66,67)$.

Several strategies targeting TAMs are under investigation including: TAM depletion, TAM exclusion from the TME, TAM reprogramming from $M 2$ to $M 1$ and restoring phagocytic capacity.

The combination of PD0360324, a monoclonal antibody against macrophages colony-stimulating factor, with cyclophosphamide is under clinical investigation for patients with HGSOC in a phase II trial (NCT02948101).

CXCR4/CXCL12 contributes to the recruitment of the suppressive M2 macrophages and has been correlated with poor clinical outcome in OC (68-70). Pharmacological inhibition of this pathway with the CXCR4 inhibitor, AMD3100, alone or in combination with anti-PD-1 therapy have shown promising results in hepatocellular and ovarian preclinical models (71).

To restore the phagocytic capacity of TAMs, one of the most investigated strategy is the inhibition of CD47/SIRP $\alpha$ pathway. A number of therapeutics that target the CD47/SIRP $\alpha$ axis are under preclinical and clinical investigation (72). A phase I trial of an antiCD47 antibody Hu5F9-G4 demonstrated encouraging results in OC, two patients with ovarian/fallopian tube cancers had partial remissions for 5.2 and 9.2 months (73). These results have led to an ongoing phase I trial testing Hu5F9-G4 in combination with avelumab in patients with OC (NCT03558139). BI 765063 is a
SIRP $\alpha$ inhibitor tested in a phase I dose escalation as monotherapy or in combination with ezabenlimab (anti PD-1) in advanced solid tumors has showed promising clinical activity, including one partial response in monotherapy (hepatocellular carcinoma) and three partial response in combination (endometrial and colorectal cancer) (74).

\section{Regulatory T Cells}

Regulatory T-cells mediate a suppressive microenvironment though the inhibition of $\mathrm{T}$-cell proliferation/recruitment, cytokine production (TGF- $\beta$, IL-10, IL-35) and suppression of antigen presentation in DC in a majority of cancers (75-77). Several studies have shown that high Foxp3 expression by Tregs is associated with poor prognosis in ovarian cancer in terms of overall survival and progression-free survival $(78,79)$.

Denileukin Difitox (Ontak) is a recombinant fusion protein product of diphtheria toxin and IL-2 that selectively binds to CD25 on Tregs and can cause their depletion. Ontak has sown promising results in melanoma and is currently being tested in OC. In a phase I clinical trial involving seven patients with advanced adenocarcinomas, including ovarian cancers, treatment with Ontak was associated with a reduction in peripheral blood CD3+/CD4+/CD25+ cells and an increase in the number of circulating IFN- $\gamma$-producing T cells (80). On this basis, a phase II trial of Ontak in OC was initiated (81).

Toll-like receptor agonist-8 (TLR8) can reverse the suppressive function of human $\mathrm{CD}^{+} 5^{+}$Treg (82-84). VTX- 
2337, a synthetic small-molecule agonist specific to TLR8 was investigated in two phase II trials in recurrent OC $(85,86)$. Unfortunately, the addition of VTX-2337 to pegylated lyposomal doxorubicin did not improve clinical outcomes compared with placebo. Another potent TLR8 agonist, DN052 inhibited tumor growth and enhanced efficacy of ICIs in vitro (87) and is currently advancing in phase 1 trials in patients with advanced solid tumors (NCT03934359).

Tumor necrosis factor receptor 2 is expressed by highly immunosuppressive Treg and thereby represents an attractive target protein. In vivo, inhibition of TNFR2 leads to OC cells death, Treg inhibition and T-cells effector expansion (88). A phase I/IIa of BI-1808, a monoclonal antibody against TNFR2, as a single agent and in combination with pembrolizumab is ongoing in patients with advanced malignancies including OC (NCT04752826).

The relevance of $\mathrm{B}$ cell population and in particular regulatory $\mathrm{B}$ cells is poorly described in OC, there are some loose correlations with outcome in retrospective studies. For example CD19+ B cell populations tend to predict poor survival while CD20+ B cells predict improved PFS. However therapeutic strategies targeting B cells are currently lacking in solid tumors, especially OC (89).

\section{Cancer-Associated Fibroblasts}

Cancer-associated fibroblasts have been implicated in tumor proliferation, invasion, metastasis, angionesis and resistance to cancer therapeutics $(90,91)$. More recently, some CAF subsets have been shown to dampen the anti-tumor immune response (92). CAFs secrete numerous cytokines including TGF- $\beta$, IL-6, IL-8, IL10 , and VEGF that contribute to the immunosuppressive TME by promoting monocyte recruitment or macrophages differentiation to M2-phenotype (93). Through the secretion of TGF- $\beta$, CAFs negatively regulate NK cells activation (94) and inhibit CD8+ T cell cytotoxic function by reducing the expression of perforin, granzyme and IFN-g $(95,96)$. CAFs also down-regulate the antigen presentation capacity of dendritic cells $(97,98)$ and increase differentiation of Tregs $(98,99)$. The capacity of CAFs to suppress anti-tumor immunity makes them another promising therapeutic target for cancer treatment. FAP (fibroblast associated protein) and $\alpha$-Smooth Muscle Actine ( $\alpha$-SMA) are markers of a particularly immunosuppressive subpopulation of CAFs.

Numerous approaches have been investigated in pre-clinical and clinical models such as CAF depletion by targeting FAP with pharmacological inhibitors, monoclonal antibodies, DNA FAP vaccines and CAR-T cells specific for FAP (100-104).

Two types of bispecific antibody targeting FAP/IL-2 (RO6874281) and FAP/4-1BB(RO7122290) are currently under investigation as CAF-targeting strategies. RO6874281 was shown to activate $\mathrm{CD}^{+} \mathrm{T}$-cells and NK cells and to reduce Treg activity (105). The bispecific antibody targeting FAP/4-1BB enhanced Tcell stimulation in vivo and led to tumor remission in mouse models (106). An ongoing phase I trial is currently testing RO7122290 in monotherapy or in combination with atezolizumab in patients with advanced solid tumors (107).

Finally one last strategy is to target a ubiquitous immunosuppressive cytokine, such as TGF- $\beta$. Downregulation of TGF- $\beta$ could inhibit TAMs, CAFs, Tregs as well many other immune tolerant subsets. Gemogenovatucel-T (Vigil) is an autologous tumor cell vaccine which specifically reduces expression of furin and downstream TGF- $\beta 1$ and TGF- $\beta 2$. Vigil was tested in a phase II trial as immunotherapy maintenance after $1^{\text {st }}$ line chemotherapy for advanced newly diagnosed OC (108). VIGIL showed a trend for an improved recurrence-free survival vs placebo (11,5 vs. 8,4 months); intriguingly, the benefit was significant among the subset with HR proficient tumors (RFS:10,6 vs 5,7mo, $\mathrm{p}=0,007$ )versus $55 \%$ for placebo, $\mathrm{p}=$ ). Combination of Vigil with atezolizumab or durvalumab is currently being tested (NCT03073525, NCT02725489).

\section{Co-Regulatory Molecules}

Immune cells express a variety of other co-regulatory molecules beyond PD-L1/PD-1 which could be targeted for the development of new immunotherapeutic strategies for patients with refractory tumors.

\section{Co-Inhibitors}

TIM-3 and LAG -3 act as negative regulators of activation and proliferation of T-cells. High expression of TIM-3 have been detected in OC and associated with poor prognosis $(109,110)$. In a study involving 98 patients with OC, TIM-3 was the most prevalent co-regulator with more than $75 \%$ of the samples being TIM-3 positive (111). Multiple Phase I clinical trials are currently testing anti-TIM-3 antibodies alone or in combination with anti PD-1 therapy for the treatment of cervical and ovarian cancer and advanced recurrent solid tumors (NCT03099109, NCT02608268, NCT03652077).

LAG-3 have been shown to play a important role in the development of OC (112). $\mathrm{CD}^{+}$lymphocytes co-expressing LAG-3 and PD-1 demonstrate impaired effector function and IFN production (113). Huang et al. found that LAG-3 and PD-1 inhibit T-cell signaling synergistically when they are coexpressed on TILs (114). The addition of the Anti-LAG-3 antibody, relatlimab significantly enhanced benefit from PD1 inhibition in a phase III trial in melanoma (115).

$\mathrm{B} 7-\mathrm{H} 3$ and $\mathrm{B} 7-\mathrm{H} 4$ are members of the immune regulatory ligand of the B7 family and both have been found to be overexpressed in OC (in $93 \%$ and $100 \%$ of the tumors, respectively) $(116,117)$. Three agents: MGD009, a dual-affinity re-targeting protein against B7-H3, DS-7300a FPA150, two antibodies targeting B7-H3 and B7-H4 respectively, are being investigated against solid tumors (NCT03406949, NCT04145622, NCT03514121).

\section{Co-Stimulators}

$4-1 \mathrm{BB}$ (CD137) is a member of the TNF receptor family and mainly expressed by activated T-cells and APC. Signaling via 4-1BB upregulates survival genes, enhances cell division, induces cytokine production, and prevents activation-induced cell death in T cells. In OC, 4-1BB has been investigated in combination with other immune checkpoint agents such as PD-1 and TIM-3. Combination of CD137 stimulation with PD-1 inhibition in mouse ovarian cancer model induce synergistic antitumor immune response. Currently, no trial is specifically targeting 4$1 \mathrm{BB}$ specifically for gynecologic tumors although multiple phase I trials are under investigation in solid tumors. 
OX40 (CD134), a member of the TNF superfamily, is mainly detected on active effector $\mathrm{CD} 4^{+} \mathrm{T}$ cell and NKT cells, as well as on Tregs. OX40 has dualistic and opposing functions depending on the cell type; it is agonist on cytotoxic T and NK cells but inhibitory on Tregs. Ramser et al. have evaluated the expression of OX40 in 47 samples of HGSOC and found that high expression of OX40 was associated with chemosensitivity and prognosis (118). Treatment with ATOR-1015, a bi-specific CTLA-4 antagonist and OX40 agonist antibody, induces T-cell activation and Treg depletion in vitro, reduces tumor growth and improves survival in syngeneic tumor models (119).

Glucocorticoid-induced tumor necrosis factor receptor (GITR) is predominately expressed on active B cells, NK cells, and T-cells. In a study conducted by M T Zhu et al., GITR expression in malignant cells was detected in $3.2 \%$ of OC (120). Combination of PD-1 blockade and GITR triggering showed promising results in murine ID8 OC, with $20 \%$ of the mice becoming tumor-free 90 days after tumor injection. Combined treatment with anti-PD-1/GITR antibody and chemotherapeutic drugs further increased the antitumor efficacy with $80 \%$ of mice achieving tumor-free long-term survival (121).

\section{CONCLUSION}

Immune checkpoint inhibitors are some of the most prominent agents that strengthens the activity of our adaptive immune

\section{REFERENCES}

1. Ferlay J, Soerjomataram I, Dikshit R, Eser S, Mathers C, Rebelo M, et al. Cancer Incidence and Mortality Worldwide: Sources, Methods and Major Patterns in GLOBOCAN 2012. Int J Cancer (2015) 136:E359-386. doi: 10.1002/ijc.29210

2. Kehoe S, Hook J, Nankivell M, Jayson GC, Kitchener H, Lopes T, et al. Primary Chemotherapy Versus Primary Surgery for Newly Diagnosed Advanced Ovarian Cancer (CHORUS): An Open-Label, Randomised, Controlled, Non-Inferiority Trial. Lancet Lond Engl (2015) 386:249-57. doi: 10.1016/S0140-6736(14)62223-6

3. Vergote I, Tropé CG, Amant F, Kristensen GB, Ehlen T, Johnson N, et al. Neoadjuvant Chemotherapy or Primary Surgery in Stage IIIC or IV Ovarian Cancer. N Engl J Med (2010) 363:943-53. doi: 10.1056/NEJMoa0908806

4. Bukowski RM, Ozols RF, Markman M. The Management of Recurrent Ovarian Cancer. Semin Oncol (2007) 34:S1-15. doi: 10.1053/ j.seminoncol.2007.03.012

5. Alsaab HO, Sau S, Alzhrani R, Tatiparti K, Bhise K, Kashaw SK, et al. PD-1 and PD-L1 Checkpoint Signaling Inhibition for Cancer Immunotherapy: Mechanism, Combinations, and Clinical Outcome. Front Pharmacol (2017) 8:561. doi: 10.3389/fphar.2017.00561

6. Wei SC, Levine JH, Cogdill AP, Zhao Y, Anang N-AAS, Andrews MC, et al. Distinct Cellular Mechanisms Underlie Anti-CTLA-4 and Anti-PD-1 Checkpoint Blockade. Cell (2017) 170:1120-33.e17. doi: 10.1016/ j.cell.2017.07.024

7. Li J, Wang J, Chen R, Bai Y, Lu X. The Prognostic Value of TumorInfiltrating T Lymphocytes in Ovarian Cancer. Oncotarget (2017) 8:1562131. doi: 10.18632/oncotarget.14919

8. Hamanishi J, Mandai M, Iwasaki M, Okazaki T, Tanaka Y, Yamaguchi K, et al. Programmed Cell Death 1 Ligand 1 and Tumor-Infiltrating CD8+ T Lymphocytes are Prognostic Factors of Human Ovarian Cancer. Proc Natl Acad Sci USA (2007) 104:3360-5. doi: 10.1073/pnas.0611533104 system, and have demonstrated success in treating different types of cancer. With significant promises in melanoma and other solid tumors, ICIs have also been evaluated in OC. Contrary to expectations, their efficacy for treating OC is very low.

OC's immunosuppressive TME may contribute to the limited activity of ICIs. Moreover, CD8+ cells and PD-L1 may not be the only relevant immune targets in OC. Targeting other immune subsets such as TAMs, Tregs or CAFs may be relevant to make progress in cancer immunotherapy. In addition to the PD-L1/ PD-1 axis, other immunosuppressive molecules, such as CTLA4, TIM-3 and LAG-3 should be taken into consideration for the development of new immunotherapeutic strategies. Finally, although not the subject of this review, other promising strategies include next generation approaches such as TCR engineering, CAR-T cells, dendritic vaccination, TILs based therapies or oncolytic viruses.

\section{AUTHOR CONTRIBUTIONS}

Both authors contributed to the conception, writing, and editing.

\section{FUNDING}

LC PhD student funded by educational grant from ARCAGY-GINECO.

9. Hwang W-T, Adams SF, Tahirovic E, Hagemann IS, Coukos G. Prognostic Significance of Tumor-Infiltrating T Cells in Ovarian Cancer: A Meta-Analysis. Gynecol Oncol (2012) 124:192-8. doi: 10.1016/ j.ygyno.2011.09.039

10. Disis ML, Taylor MH, Kelly K, Beck JT, Gordon M, Moore KM, et al. Efficacy and Safety of Avelumab for Patients With Recurrent or Refractory Ovarian Cancer: Phase 1b Results From the JAVELIN Solid Tumor Trial. JAMA Oncol (2019) 5:393-401. doi: 10.1001/ jamaoncol.2018.6258

11. Liu JF, Gordon M, Veneris J, Braiteh F, Balmanoukian A, Eder JP, et al. Safety, Clinical Activity and Biomarker Assessments of Atezolizumab From a Phase I Study in Advanced/Recurrent Ovarian and Uterine Cancers. Gynecol Oncol (2019) 154:314-22. doi: 10.1016/j.ygyno.2019.05.021

12. Varga A, Piha-Paul S, Ott PA, Mehnert JM, Berton-Rigaud D, Morosky A, et al. Pembrolizumab in Patients With Programmed Death Ligand 1-Positive Advanced Ovarian Cancer: Analysis of KEYNOTE-028. Gynecol Oncol (2019) 152:243-50. doi: 10.1016/j.ygyno.2018.11.017

13. Matulonis UA, Shapira-Frommer R, Santin AD, Lisyanskaya AS, Pignata S, Vergote I, et al. Antitumor Activity and Safety of Pembrolizumab in Patients With Advanced Recurrent Ovarian Cancer: Results From the Phase II KEYNOTE-100 Study. Ann Oncol Off J Eur Soc Med Oncol (2019) 30:1080-7. doi: 10.1093/annonc/mdz135

14. Hamanishi J, Mandai M, Ikeda T, Minami M, Kawaguchi A, Murayama T, et al. Safety and Antitumor Activity of Anti-PD-1 Antibody, Nivolumab, in Patients With Platinum-Resistant Ovarian Cancer. J Clin Oncol Off J Am Soc Clin Oncol (2015) 33:4015-22. doi: 10.1200/JCO.2015.62.3397

15. Davis AA, Patel VG. The Role of PD-L1 Expression as a Predictive Biomarker: An Analysis of All US Food and Drug Administration (FDA) Approvals of Immune Checkpoint Inhibitors. J Immunother Cancer (2019) 7:278. doi: 10.1186/s40425-019-0768-9

16. Moore KN, Okamoto A, Wu F, Lin YG, Pignata S. IMagyn050 / GOG3015 / ENGOT-Ov39: A Randomized, Double-Blind, Phase III Study of 
Atezolizumab vs Placebo Combined With Chemotherapy + Bevacizumab in Stage III-IV Ovarian, Fallopian Tube \& Peritoneal Cancers (OC). Ann Oncol (2017) 28:v350-1. doi: 10.1093/annonc/mdx372.056

17. Yu H, Boyle TA, Zhou C, Rimm DL, Hirsch FR. PD-L1 Expression in Lung Cancer. J Thorac Oncol (2016) 11:964-75. doi: 10.1016/ j.jtho.2016.04.014

18. Goodman AM, Kato S, Bazhenova L, Patel SP, Frampton GM, Miller V, et al. Tumor Mutational Burden as an Independent Predictor of Response to Immunotherapy in Diverse Cancers. Mol Cancer Ther (2017) 16:2598-608. doi: 10.1158/1535-7163.MCT-17-0386

19. Riviere P, Goodman AM, Okamura R, Barkauskas DA, Whitchurch TJ, Lee $\mathrm{S}$, et al. High Tumor Mutational Burden Correlates With Longer Survival in Immunotherapy-Naïve Patients With Diverse Cancers. Mol Cancer Ther (2020) 19:2139-45. doi: 10.1158/1535-7163.MCT-20-0161

20. Strickler JH, Hanks BA, Khasraw M. Tumor Mutational Burden as a Predictor of Immunotherapy Response: Is More Always Better? Clin Cancer Res (2021) 27:1236-41. doi: 10.1158/1078-0432.CCR-20-3054

21. Rizvi NA, Hellmann MD, Snyder A, Kvistborg P, Makarov V, Havel JJ, et al. Cancer Immunology. Mutational Landscape Determines Sensitivity to PD-1 Blockade in non-Small Cell Lung Cancer. Science (2015) 348:124-8. doi: $10.1126 /$ science.aaal 348

22. Vareki SM. High and Low Mutational Burden Tumors Versus Immunologically Hot and Cold Tumors and Response to Immune Checkpoint Inhibitors. J Immunother Cancer (2018) 6:157. doi: 10.1186/ s40425-018-0479-7

23. Fan S, Gao X, Qin Q, Li H, Yuan Z, Zhao S. Association Between Tumor Mutation Burden and Immune Infiltration in Ovarian Cancer. Int Immunopharmacol (2020) 89:107126. doi: 10.1016/j.intimp.2020.107126

24. Martin SD, Brown SD, Wick DA, Nielsen JS, Kroeger DR, Twumasi-Boateng $\mathrm{K}$, et al. Low Mutation Burden in Ovarian Cancer May Limit the Utility of Neoantigen-Targeted Vaccines. PloS One (2016) 11:e0155189. doi: 10.1371/ journal.pone. 0155189

25. Strickland KC, Howitt BE, Shukla SA, Rodig S, Ritterhouse LL, Liu JF, et al. Association and Prognostic Significance of BRCA1/2-Mutation Status With Neoantigen Load, Number of Tumor-Infiltrating Lymphocytes and Expression of PD-1/PD-L1 in High Grade Serous Ovarian Cancer. Oncotarget (2016) 7:13587-98. doi: 10.18632/oncotarget.7277

26. Rodriguez GM, Galpin KJC, McCloskey CW, Vanderhyden BC. The Tumor Microenvironment of Epithelial Ovarian Cancer and Its Influence on Response to Immunotherapy. Cancers (2018) 10:242. doi: 10.3390/ cancers 10080242

27. Manchana T, Phoolcharoen N, Tantbirojn P. BRCA Mutation in High Grade Epithelial Ovarian Cancers. Gynecol Oncol Rep (2019) 29:102-5. doi: 10.1016/j.gore.2019.07.007

28. The Cancer Genome Atlas Research Network. Integrated Genomic Analyses of Ovarian Carcinoma. Nature (2011) 474:609-15. doi: 10.1038/nature10166

29. Hao D, Liu J, Chen M, Li J, Wang L, Li X, et al. Immunogenomic Analyses of Advanced Serous Ovarian Cancer Reveal Immune Score is a Strong Prognostic Factor and an Indicator of Chemosensitivity. Clin Cancer Res Off J Am Assoc Cancer Res (2018) 24:3560-71. doi: 10.1158/1078-0432.CCR$17-3862$

30. Ledermann JA, Colombo N, Oza AM, Fujiwara K, Birrer MJ, Randall LM, et al. Avelumab in Combination With and/or Following Chemotherapy vs Chemotherapy Alone in Patients With Previously Untreated Epithelial Ovarian Cancer: Results From the Phase 3 Javelin Ovarian 100 Trial. Gynecol Oncol (2020) 159:13-4. doi: 10.1016/j.ygyno.2020.06.025

31. Li T, Chen ZJ. The cGAS-cGAMP-STING Pathway Connects DNA Damage to Inflammation, Senescence, and Cancer. J Exp Med (2018) 215:1287-99. doi: 10.1084/jem.20180139

32. Ding L, Kim H-J, Wang Q, Kearns M, Jiang T, Ohlson CE, et al. PARP Inhibition Elicits STING-Dependent Antitumor Immunity in Brca1Deficient Ovarian Cancer. Cell Rep (2018) 25:2972-2980.e5. doi: 10.1016/ j.celrep.2018.11.054

33. Drew Y, Kaufman B, Banerjee S, Lortholary A, Hong SH, Park YH, et al. Phase II Study of Olaparib + Durvalumab (MEDIOLA): Updated Results in Germline BRCA-Mutated Platinum-Sensitive Relapsed (PSR) Ovarian Cancer (OC). Ann Oncol (2019) 30:v485-6. doi: 10.1093/annonc/ mdz253.016
34. Konstantinopoulos PA, Waggoner S, Vidal GA, Mita M, Moroney JW, Holloway R, et al. Single-Arm Phases 1 and 2 Trial of Niraparib in Combination With Pembrolizumab in Patients With Recurrent PlatinumResistant Ovarian Carcinoma. JAMA Oncol (2019) 5:1141-9. doi: 10.1001/ jamaoncol.2019.1048

35. Carmeliet P. VEGF as a Key Mediator of Angiogenesis in Cancer. Oncology (2005) 69 Suppl 3:4-10. doi: 10.1159/000088478

36. Mukherjee S, Pal M, Mukhopadhyay S, Das I, Hazra R, Ghosh S, et al. VEGF Expression to Support Targeted Therapy in Ovarian Surface Epithelial Neoplasms. J Clin Diagn Res JCDR (2017) 11:EC43-6. doi: 10.7860/JCDR/ 2017/24670.9737

37. Masoumi Moghaddam S, Amini A, Morris DL, Pourgholami MH. Significance of Vascular Endothelial Growth Factor in Growth and Peritoneal Dissemination of Ovarian Cancer. Cancer Metastasis Rev (2012) 31:143-62. doi: 10.1007/s10555-011-9337-5

38. Lapeyre-Prost A, Terme M, Pernot S, Pointet A-L, Voron T, Tartour E, et al. Immunomodulatory Activity of VEGF in Cancer. Int Rev Cell Mol Biol (2017) 330:295-342. doi: 10.1016/bs.ircmb.2016.09.007

39. Martino E, Misso G, Pastina P, Costantini S, Vanni F, Gandolfo C, et al. Immune-Modulating Effects of Bevacizumab in Metastatic non-Small-Cell Lung Cancer Patients. Cell Death Discov (2016) 2:16025. doi: 10.1038/ cddiscovery.2016.25

40. Voron T, Colussi O, Marcheteau E, Pernot S, Nizard M, Pointet A-L, et al. VEGF-A Modulates Expression of Inhibitory Checkpoints on CD8+ T Cells in Tumors. J Exp Med (2015) 212:139-48. doi: 10.1084/jem.20140559

41. Liu JF, Herold C, Gray KP, Penson RT, Horowitz N, Konstantinopoulos PA, et al. Assessment of Combined Nivolumab and Bevacizumab in Relapsed Ovarian Cancer: A Phase 2 Clinical Trial. JAMA Oncol (2019) 5:1731-8. doi: 10.1001/jamaoncol.2019.3343

42. Lwin Z, Gomez-Roca C, Saada-Bouzid E, Yanez E, Muñoz FL, Im S-A, et al. LBA41 LEAP-005: Phase II Study of Lenvatinib (Len) Plus Pembrolizumab (Pembro) in Patients (Pts) With Previously Treated Advanced Solid Tumours. Ann Oncol (2020) 31:S1170. doi: 10.1016/j.annonc.2020.08.2271

43. Bracci L, Schiavoni G, Sistigu A, Belardelli F. Immune-Based Mechanisms of Cytotoxic Chemotherapy: Implications for the Design of Novel and Rationale-Based Combined Treatments Against Cancer. Cell Death Differ (2014) 21:15-25. doi: 10.1038/cdd.2013.67

44. Park YH, Lal S, Lee JE, Choi Y-L, Wen J, Ram S, et al. Chemotherapy Induces Dynamic Immune Responses in Breast Cancers That Impact Treatment Outcome. Nat Commun (2020) 11:6175. doi: 10.1038/s41467-020-19933-0

45. Opzoomer JW, Sosnowska D, Anstee JE, Spicer JF, Arnold JN. Cytotoxic Chemotherapy as an Immune Stimulus: A Molecular Perspective on Turning Up the Immunological Heat on Cancer. Front Immunol (2019) 10:1654. doi: 10.3389/fimmu.2019.01654

46. Hannani D, Sistigu A, Kepp O, Galluzzi L, Kroemer G, Zitvogel L. Prerequisites for the Antitumor Vaccine-Like Effect of Chemotherapy and Radiotherapy. Cancer J Sudbury Mass (2011) 17:351-8. doi: 10.1097/ PPO.0b013e3182325d4d

47. Pol J, Vacchelli E, Aranda F, Castoldi F, Eggermont A, Cremer I, et al. Trial Watch: Immunogenic Cell Death Inducers for Anticancer Chemotherapy. Oncoimmunology (2015) 4:e1008866. doi: 10.1080/2162402X.2015.1008866

48. Pujade-Lauraine E, Fujiwara K, Ledermann JA, Oza AM, Kristeleit RS, RayCoquard IL, et al. Avelumab Alone or in Combination With Pegylated Liposomal Doxorubicin Versus Pegylated Liposomal Doxorubicin Alone in Platinum-Resistant or Refractory Epithelial Ovarian Cancer: Primary and Biomarker Analysis of the Phase III JAVELIN Ovarian 200 Trial. Gynecol Oncol (2019) 154:21-2. doi: 10.1016/j.ygyno.2019.04.053

49. Zamarin D, Burger RA, Sill MW, Powell DJ, Lankes HA, Feldman MD, et al. Randomized Phase II Trial of Nivolumab Versus Nivolumab and Ipilimumab for Recurrent or Persistent Ovarian Cancer: An NRG Oncology Study. J Clin Oncol Off J Am Soc Clin Oncol (2020) 38:1814-23. doi: 10.1200/JCO.19.02059

50. Leary A, de la Motte Rouge T, Lortholary A, Asselain B, Alexandre J, Floquet A, et al. 727p Phase Ib INEOV Neoadjuvant Trial of the Anti-PDL1, Durvalumab (D) +/- Anti-CTLA4 Tremelimumab (T) With Platinum Chemotherapy for Patients (Pts) With Unresectable Ovarian Cancer (OC): A GINECO Study. Ann Oncol (2021) 32:S731. doi: 10.1016/ j.annonc.2021.08.1170 
51. Takaishi K, Komohara Y, Tashiro H, Ohtake H, Nakagawa T, Katabuchi H, et al. Involvement of M2-Polarized Macrophages in the Ascites From Advanced Epithelial Ovarian Carcinoma in Tumor Progression via Stat3 Activation. Cancer Sci (2010) 101:2128-36. doi: 10.1111/j.13497006.2010.01652.x

52. Colvin EK. Tumor-Associated Macrophages Contribute to Tumor Progression in Ovarian Cancer. Front Oncol (2014) 4:137. doi: 10.3389/ fonc.2014.00137

53. Nowak M, Klink M. The Role of Tumor-Associated Macrophages in the Progression and Chemoresistance of Ovarian Cancer. Cells (2020) 9:1299. doi: 10.3390/cells9051299

54. Hensler M, Kasikova L, Fiser K, Rakova J, Skapa P, Laco J, et al. M2-Like Macrophages Dictate Clinically Relevant Immunosuppression in Metastatic Ovarian Cancer. J Immunother Cancer (2020) 8:e000979. doi: 10.1136/jitc2020-000979

55. Cheng H, Wang Z, Fu L, Xu T. Macrophage Polarization in the Development and Progression of Ovarian Cancers: An Overview. Front Oncol (2019) 9:421. doi: 10.3389/fonc.2019.00421

56. Reinartz S, Schumann T, Finkernagel F, Wortmann A, Jansen JM, Meissner W, et al. Mixed-Polarization Phenotype of Ascites-Associated Macrophages in Human Ovarian Carcinoma: Correlation of CD163 Expression, Cytokine Levels and Early Relapse. Int J Cancer (2014) 134:32-42. doi: 10.1002/ ijc. 28335

57. Lan C, Huang X, Lin S, Huang H, Cai Q, Wan T, et al. Expression of M2Polarized Macrophages is Associated With Poor Prognosis for Advanced Epithelial Ovarian Cancer. Technol Cancer Res Treat (2013) 12:259-67. doi: $10.7785 /$ tcrt.2012.500312

58. Zhang M, He Y, Sun X, Li Q, Wang W, Zhao A, et al. A High M1/M2 Ratio of Tumor-Associated Macrophages Is Associated With Extended Survival in Ovarian Cancer Patients. J Ovarian Res (2014) 7:19. doi: 10.1186/1757-2215-7-19

59. Pan Y, Yu Y, Wang X, Zhang T. Tumor-Associated Macrophages in Tumor Immunity. Front Immunol (2020) 11:583084. doi: 10.3389/ fimmu.2020.583084

60. Yang Q, Guo N, Zhou Y, Chen J, Wei Q, Han M. The Role of TumorAssociated Macrophages (TAMs) in Tumor Progression and Relevant Advance in Targeted Therapy. Acta Pharm Sin B (2020) 10:2156-70. doi: 10.1016/j.apsb.2020.04.004

61. Noy R, Pollard JW. Tumor-Associated Macrophages: From Mechanisms to Therapy. Immunity (2014) 41:49-61. doi: 10.1016/j.immuni.2014.06.010

62. Massi D, Marconi C, Franchi A, Bianchini F, Paglierani M, Ketabchi S, et al. Arginine Metabolism in Tumor-Associated Macrophages in Cutaneous Malignant Melanoma: Evidence From Human and Experimental Tumors. Hum Pathol (2007) 38:1516-25. doi: 10.1016/j.humpath.2007.02.018

63. Grzywa TM, Sosnowska A, Matryba P, Rydzynska Z, Jasinski M, Nowis D, et al. Myeloid Cell-Derived Arginase in Cancer Immune Response. Front Immunol (2020) 11:938. doi: 10.3389/fimmu.2020.00938

64. Arlauckas SP, Garren SB, Garris CS, Kohler RH, Oh J, Pittet MJ, et al. Arg1 Expression Defines Immunosuppressive Subsets of Tumor-Associated Macrophages. Theranostics (2018) 8:5842-54. doi: 10.7150/thno.26888

65. Matlung HL, Szilagyi K, Barclay NA, van den Berg TK. The CD47-Sirp $\alpha$ Signaling Axis as an Innate Immune Checkpoint in Cancer. Immunol Rev (2017) 276:145-64. doi: 10.1111/imr.12527

66. Willingham SB, Volkmer J-P, Gentles AJ, Sahoo D, Dalerba P, Mitra SS, et al. The CD47-Signal Regulatory Protein Alpha (SIRPa) Interaction Is a Therapeutic Target for Human Solid Tumors. Proc Natl Acad Sci USA (2012) 109:6662-7. doi: 10.1073/pnas.1121623109

67. Liu R, Wei H, Gao P, Yu H, Wang K, Fu Z, et al. CD47 Promotes Ovarian Cancer Progression by Inhibiting Macrophage Phagocytosis. Oncotarget (2017) 8:39021-32. doi: 10.18632/oncotarget.16547

68. Liu C-F, Liu S-Y, Min X-Y, Ji Y-Y, Wang N, Liu D, et al. The Prognostic Value of CXCR4 in Ovarian Cancer: A Meta-Analysis. PloS One (2014) 9: e92629. doi: 10.1371/journal.pone.0092629

69. Popple A, Durrant LG, Spendlove I, Rolland P, Scott IV, Deen S, et al. The Chemokine, CXCL12, Is an Independent Predictor of Poor Survival in Ovarian Cancer. Br J Cancer (2012) 106:1306-13. doi: 10.1038/bjc.2012.49

70. Mao TL, Fan KF, Liu CL. Targeting the CXCR4/CXCL12 Axis in Treating Epithelial Ovarian Cancer. Gene Ther (2017) 24:621-9. doi: 10.1038/ gt.2017.69
71. Zeng Y, Li B, Liang Y, Reeves PM, Qu X, Ran C, et al. Dual Blockade of CXCL12-CXCR4 and PD-1-PD-L1 Pathways Prolongs Survival of Ovarian Tumor-Bearing Mice by Prevention of Immunosuppression in the Tumor Microenvironment. FASEB J (2019) 33:6596-608. doi: 10.1096/ fj.201802067RR

72. Kaur S, Cicalese KV, Banerjee R, Roberts DD. Preclinical and Clinical Development of Therapeutic Antibodies Targeting Functions of CD47 in the Tumor Microenvironment. Antib Ther (2020) 3:179-92. doi: 10.1093/abt/ tbaa017

73. Sikic BI, Lakhani N, Patnaik A, Shah SA, Chandana SR, Rasco D, et al. FirstIn-Human, First-In-Class Phase I Trial of the Anti-CD47 Antibody Hu5F9G4 in Patients With Advanced Cancers. J Clin Oncol (2019) 37:946-53. doi: 10.1200/JCO.18.02018

74. Kotecki N, Champiat S, Delord J-P, Vinceneux A, Jungels C, Marabelle A, et al. 983p Phase I Dose Escalation Study in Patients (Pts) With Advanced Solid Tumours Receiving First-in-Class BI 765063, a Selective SignalRegulatory Protein $\alpha(\operatorname{Sirp} \alpha)$ Inhibitor, in Combination With Ezabenlimab (BI 754091), a Programmed Cell Death Protein 1 (PD-1) Inhibitor. Ann Oncol (2021) 32:S841-2. doi: 10.1016/j.annonc.2021.08.1367

75. Paluskievicz CM, Cao X, Abdi R, Zheng P, Liu Y. Bromberg JS. T Regulatory Cells and Priming the Suppressive Tumor Microenvironment. Front Immunol (2019) 10:2453. doi: 10.3389/fimmu.2019.02453

76. Li C, Jiang P, Wei S, Xu X, Wang J. Regulatory T Cells in Tumor Microenvironment: New Mechanisms, Potential Therapeutic Strategies and Future Prospects. Mol Cancer (2020) 19:116. doi: 10.1186/s12943-020-01234-1

77. Togashi Y, Shitara K, Nishikawa H. Regulatory T Cells in Cancer Immunosuppression - Implications for Anticancer Therapy. Nat Rev Clin Oncol (2019) 16:356-71. doi: 10.1038/s41571-019-0175-7

78. Curiel TJ, Coukos G, Zou L, Alvarez X, Cheng P, Mottram P., et al Specific Recruitment of Regulatory T Cells in Ovarian Carcinoma Fosters Immune Privilege and Predicts Reduced Survival. Nat Med (2004) 10:942-9. doi: 10.1038/nm1093

79. Wolf D, Wolf AM, Rumpold H, Fiegl H, Zeimet AG, Muller-Holzner E, et al. The Expression of the Regulatory T Cell-Specific Forkhead Box Transcription Factor FoxP3 is Associated With Poor Prognosis in Ovarian Cancer. Clin Cancer Res Off J Am Assoc Cancer Res (2005) 11:8326-31. doi: 10.1158/1078-0432.CCR-05-1244

80. Barnett B, Kryczek I, Cheng P, Zou W, Curiel TJ. Regulatory T Cells in Ovarian Cancer: Biology and Therapeutic Potential. Am J Reprod Immunol (2005) 54(6):369-77. doi: 10.1111/j.1600-0897.2005.00330.x

81. Curiel TJ, Coukos G, Zou L, Alvarez X, Cheng P, Mottram P, et al. Specific Recruitment of Regulatory T Cells in Ovarian Carcinoma Fosters Immune Privilege and Predicts Reduced Survival. Nat Med (2004) 10(9):942-9. doi: 10.1038/nm1093

82. Xu R, Wu M, Liu S, Shang W, Li R, Xu J, et al. Glucose Metabolism Characteristics and TLR8-Mediated Metabolic Control of CD4+ Treg Cells in Ovarian Cancer Cells Microenvironment. Cell Death Dis (2021) 12:1-11. doi: 10.1038/s41419-020-03272-5

83. Peng G, Guo Z, Kiniwa Y, Voo KS, Peng W, Fu T, et al. Toll-Like Receptor 8Mediated Reversal of CD4+ Regulatory T Cell Function. Science (2005) 309:1380-4. doi: 10.1126/science.1113401

84. Liu X, Li L, Peng G. TLR8 Reprograms Human Treg Metabolism and Function. Aging (2019) 11:6614-5. doi: 10.18632/aging.102223

85. Monk BJ, Facciabene A, Brady WE, Aghajanian CA, Fracasso PM, Walker JL, et al. Integrative Development of a TLR8 Agonist for Ovarian Cancer Chemoimmunotherapy. Clin Cancer Res Off J Am Assoc Cancer Res (2017) 23:1955-66. doi: 10.1158/1078-0432.CCR-16-1453

86. Monk BJ, Brady MF, Aghajanian C, Lankes HA, Rizack T, Leach J, et al. A Phase 2, Randomized, Double-Blind, Placebo- Controlled Study of ChemoImmunotherapy Combination Using Motolimod With Pegylated Liposomal Doxorubicin in Recurrent or Persistent Ovarian Cancer: A Gynecologic Oncology Group Partners Study. Ann Oncol Off J Eur Soc Med Oncol (2017) 28:996-1004. doi: 10.1093/annonc/mdx049

87. Wang Y, Yang H, Li H, Zhao S, Zeng Y, Zhang P, et al. Development of A Novel Highly Selective TLR8 Agonist for Cancer Immunotherapy. Mol Biomed (2020) 1:6. doi: 10.1101/2020.03.14.991760

88. Torrey H, Butterworth J, Mera T, Okubo Y, Wang L, Baum D, et al. Targeting TNFR2 With Antagonistic Antibodies Inhibits Proliferation of 


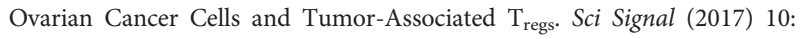
eaaf8608. doi: 10.1126/scisignal.aaf8608

89. Gupta P, Chen C, Chaluvally-Raghavan P, Pradeep S. B Cells as an ImmuneRegulatory Signature in Ovarian Cancer. Cancers (2019) 11:894. doi: $10.3390 /$ cancers 11070894

90. Kalluri R. The Biology and Function of Fibroblasts in Cancer. Nat Rev Cancer (2016) 16:582-98. doi: 10.1038/nrc.2016.73

91. Ping Q, Yan R, Cheng X, Wang W, Zhong Y, Hou Z, et al. Cancer-Associated Fibroblasts: Overview, Progress, Challenges, and Directions. Cancer Gene Ther (2021) 28:984-99. doi: 10.1038/s41417-021-00318-4

92. Kieffer Y, Hocine HR, Gentric G, Pelon F, Bernard C, Bourachot B, et al. Single-Cell Analysis Reveals Fibroblast Clusters Linked to Immunotherapy Resistance in Cancer. Cancer Discov (2020) 10:1330-51. doi: 10.1158/21598290.CD-19-1384

93. Takahashi H, Sakakura K, Kudo T, Toyoda M, Kaira K, Oyama T, et al. Cancer-Associated Fibroblasts Promote an Immunosuppressive Microenvironment Through the Induction and Accumulation of Protumoral Macrophages. Oncotarget (2017) 8:8633-47. doi: 10.18632/ oncotarget.14374

94. Donatelli SS, Zhou J-M, Gilvary DL, Eksioglu EA, Chen X, Cress WD, et al. TGF- $\beta$-Inducible microRNA-183 Silences Tumor-Associated Natural Killer Cells. Proc Natl Acad Sci USA (2014) 111:4203-8. doi: 10.1073/ pnas. 1319269111

95. Ahmadzadeh M, Rosenberg SA. TGF-Beta 1 Attenuates the Acquisition and Expressi7on of Effector Function by Tumor Antigen-Specific Human Memory CD8 T Cells. J Immunol Baltim Md 1950 (2005) 174:5215-23. doi: 10.4049/jimmunol.174.9.5215

96. Thomas DA, Massague J. TGF-Beta Directly Targets Cytotoxic T Cell Functions During Tumor Evasion of Immune Surveillance. Cancer Cell (2005) 8:369-80. doi: 10.1016/j.ccr.2005.10.012

97. Flavell RA, Sanjabi S, Wrzesinski SH, Licona-Limón P. The Polarization of Immune Cells in the Tumour Environment by Tgf $\beta$. Nat Rev Immunol (2010) 10:554-67. doi: 10.1038/nri2808

98. Cheng J-T, Deng Y-N, Yi H-M, Wang G-Y, Fu B-S, Chen W-J, et al. Hepatic Carcinoma-Associated Fibroblasts Induce IDO-Producing Regulatory Dendritic Cells Through IL-6-Mediated STAT3 Activation. Oncogenesis (2016) 5:e198-8. doi: 10.1038/oncsis.2016.7

99. Givel A-M, Kieffer Y, Scholer-Dahirel A, Sirven P, Cardon M, Pelon F, et al. Mir200-Regulated CXCL12 $\beta$ Promotes Fibroblast Heterogeneity and Immunosuppression in Ovarian Cancers. Nat Commun (2018) 9:1056. doi: 10.1038/s41467-018-03348-z

100. Kakarla S, Chow KKH, Mata M, Shaffer DR, Song X-T, Wu M-F, et al. Antitumor Effects of Chimeric Receptor Engineered Human T Cells Directed to Tumor Stroma. Mol Ther J Am Soc Gene Ther (2013) 21:1611-20. doi: $10.1038 / \mathrm{mt} .2013 .110$

101. Wang L-CS, Lo A, Scholler J, Sun J, Majumdar RS, Kapoor V, et al. Targeting Fibroblast Activation Protein in Tumor Stroma With Chimeric Antigen Receptor T Cells can Inhibit Tumor Growth and Augment Host Immunity Without Severe Toxicity. Cancer Immunol Res (2014) 2:154-66. doi: 10.1158/ 2326-6066.CIR-13-0027

102. Freedman JD, Duffy MR, Lei-Rossmann J, Muntzer A, Scott EM, Hagel J, et al. An Oncolytic Virus Expressing a T-Cell Engager Simultaneously Targets Cancer and Immunosuppressive Stromal Cells. Cancer Res (2018) 78:6852-65. doi: 10.1158/0008-5472.CAN-18-1750

103. Fabre M, Ferrer C, Domínguez-Hormaetxe S, Bockorny B, Murias L, Seifert O, et al. OMTX705, a Novel FAP-Targeting ADC Demonstrates Activity in Chemotherapy and Pembrolizumab-Resistant Solid Tumor Models. Clin Cancer Res (2020) 26:3420-30. doi: 10.1158/1078-0432.CCR-19-2238

104. Brennen WN, Isaacs JT, Denmeade SR. Rationale Behind Targeting Fibroblast Activation Protein-Expressing Carcinoma-Associated Fibroblasts as a Novel Chemotherapeutic Strategy. Mol Cancer Ther (2012) 11:257-66. doi: 10.1158/1535-7163.MCT-11-0340

105. Melero I, Alvarez EC, Mau-Sorensen M, Lassen UN, Lolkema MP, Robbrecht DG, et al. Clinical Activity, Safety, and PK/PD From a Phase I Study of RO6874281, a Fibroblast Activation Protein (FAP) Targeted Interleukin-2 Variant (IL-2v). Ann Oncol (2018) 29:viii134-viii135. doi: 10.1093/annonc/mdy279.400
106. Trüb M, Uhlenbrock F, Claus C, Herzig P, Thelen M, Karanikas V, et al. Fibroblast Activation Protein-Targeted-4-1BB Ligand Agonist Amplifies Effector Functions of Intratumoral $\mathrm{T}$ Cells in Human Cancer. J Immunother Cancer (2020) 8:e000238. doi: 10.1136/jitc-2019-000238

107. Melero I, Sanmamed MF, Calvo E, Moreno I, Moreno V, Guerrero TCH, et al. 1025mo First-In-Human (FIH) Phase I Study of RO7122290 (RO), a Novel FAP-Targeted 4-1BB Agonist, Administered as Single Agent and in Combination With Atezolizumab (ATZ) to Patients With Advanced Solid Tumours. Ann Oncol (2020) 31:S707. doi: 10.1016/ j.annonc.2020.08.1145

108. Rocconi RP, Grosen EA, Ghamande SA, Chan JK, Barve MA, Oh J, et al. Gemogenovatucel-T (Vigil) Immunotherapy as Maintenance in Frontline Stage III/IV Ovarian Cancer (VITAL): A Randomised, Double-Blind, Placebo-Controlled, Phase 2b Trial. Lancet Oncol (2020) 21:1661-72. doi: 10.1016/S1470-2045(20)30533-7

109. Xu Y, Zhang H, Huang Y, Rui X, Zheng F. Role of TIM-3 in Ovarian Cancer. Clin Transl Oncol Off Publ Fed Span Oncol Soc Natl Cancer Inst Mex (2017) 19:1079-83. doi: 10.1007/s12094-017-1656-8

110. Fucikova J, Rakova J, Hensler M, Kasikova L, Belicova L, Hladikova K, et al. TIM-3 Dictates Functional Orientation of the Immune Infiltrate in Ovarian Cancer. Clin Cancer Res (2019) 25:4820-31. doi: 10.1158/1078-0432.CCR$18-4175$

111. Blanc-Durand F, Genestie C, Galende EY, Gouy S, Morice P, Pautier P, et al. Distribution of Novel Immune-Checkpoint Targets in Ovarian Cancer Tumor Microenvironment: A Dynamic Landscape. Gynecol Oncol (2021) 160:279-84. doi: 10.1016/j.ygyno.2020.09.045

112. Long L, Zhang X, Chen F, Pan Q, Phiphatwatchara P, Zeng Y, et al. The Promising Immune Checkpoint LAG-3: From Tumor Microenvironment to Cancer Immunotherapy. Genes Cancer (2018) 9:176-89. doi: 10.18632/ genesandcancer. 180

113. Matsuzaki J, Gnjatic S, Mhawech-Fauceglia P, Beck A, Miller A, Tsuji T, et al. Tumor-Infiltrating NY-ESO-1-Specific CD8+ T Cells are Negatively Regulated by LAG-3 and PD-1 in Human Ovarian Cancer. Proc Natl Acad Sci USA (2010) 107:7875-80. doi: 10.1073/pnas.1003345107

114. Huang R-Y, Eppolito C, Lele S, Shrikant P, Matsuzaki J, Odunsi K. LAG3 and PD1 Co-Inhibitory Molecules Collaborate to Limit CD8+ T Cell Signaling and Dampen Antitumor Immunity in a Murine Ovarian Cancer Model. Oncotarget (2015) 6:27359-77. doi: 10.18632/oncotarget.4751

115. Lipson EJ, Tawbi HA-H, Schadendorf D, Ascierto PA, Matamala L, Gutiérrez EC, et al. Relatlimab (RELA) Plus Nivolumab (NIVO) Versus NIVO in First-Line Advanced Melanoma: Primary Phase III Results From RELATIVITY-047 (CA224-047). J Clin Oncol (2021) 39:9503-3. doi: 10.1200/JCO.2021.39.15_suppl.9503

116. Fauci JM, Straughn JM, Ferrone S. Buchsbaum DJ. A Review of B7-H3 and B7-H4 Immune Molecules and Their Role in Ovarian Cancer. Gynecol Oncol (2012) 127:420-5. doi: 10.1016/j.ygyno.2012.08.017

117. Zang X, Sullivan PS, Soslow RA, Waitz R, Reuter VE, Wilton A, et al. Tumor Associated Endothelial Expression of B7-H3 Predicts Survival in Ovarian Carcinomas. Mod Pathol Off J U S Can Acad Pathol Inc (2010) 23:1104-12. doi: 10.1038/modpathol.2010.95

118. Ramser M, Eichelberger S, Däster S, Weixler B, Kraljević M, Mechera R, et al. High OX40 Expression in Recurrent Ovarian Carcinoma is Indicative for Response to Repeated Chemotherapy. BMC Cancer (2018) 18:425. doi: $10.1186 / \mathrm{s} 12885-018-4339-0$

119. Kvarnhammar AM, Veitonmäki N, Hägerbrand K, Dahlman A, Smith KE, Fritzell S, et al. The CTLA-4 X OX40 Bispecific Antibody ATOR-1015 Induces Anti-Tumor Effects Through Tumor-Directed Immune Activation. J Immunother Cancer (2019) 7:103. doi: 10.1186/ s40425-019-0570-8

120. Zhu MMT, Burugu S, Gao D, Yu J, Kos Z, Leung S, et al. Evaluation of Glucocorticoid-Induced TNF Receptor (GITR) Expression in Breast Cancer and Across Multiple Tumor Types. Mod Pathol Off J U S Can Acad Pathol Inc (2020) 33:1753-63. doi: 10.1038/s41379-020-0550-z

121. Lu L, Xu X, Zhang B, Zhang R, Ji H, Wang X. Combined PD-1 Blockade and GITR Triggering Induce a Potent Antitumor Immunity in Murine Cancer Models and Synergizes With Chemotherapeutic Drugs. J Transl Med (2014) 12:36. doi: 10.1186/1479-5876-12-36 
Conflict of Interest: AL: fees to institution for ad boards with AZ, Clovis, MSD, GSK, Ability, Merck Serono. AL: support to institution for clinical trials from Roche, GSK, AZ, Agenus, Iovance, Pfizer, MSD, Incyte.

The remaining author declares that the research was conducted in the absence of any commercial or financial relationships that could be construed as a potential conflict of interest.

Publisher's Note: All claims expressed in this article are solely those of the authors and do not necessarily represent those of their affiliated organizations, or those of the publisher, the editors and the reviewers. Any product that may be evaluated in this article, or claim that may be made by its manufacturer, is not guaranteed or endorsed by the publisher.

Copyright (C) 2021 Chardin and Leary. This is an open-access article distributed under the terms of the Creative Commons Attribution License (CC BY). The use, distribution or reproduction in other forums is permitted, provided the original author(s) and the copyright owner(s) are credited and that the original publication in this journal is cited, in accordance with accepted academic practice. No use, distribution or reproduction is permitted which does not comply with these terms. 